\title{
Lower limbs muscle activation during instep kick in soccer: effects of dominance and ball condition
}

\begin{tabular}{|r|l|}
\hline Journal: & Science and Medicine in Football \\
\hline Manuscript ID & RSMF-2020-0260.R1 \\
\hline Manuscript Type: & Original Papers \\
\hline Keywords: & kicking, EMG, moving ball, sports motion \\
\hline & $\begin{array}{l}\text { Muscle activation during soccer kicking has been studied exclusively with } \\
\text { players kicking stationary balls with the dominant foot. This study } \\
\text { evaluated swinging and support limb muscle activation during the same } \\
\text { kind of kick (instep) using different feet and ball approach conditions. } \\
\text { Vastus medialis (VM), biceps femoris (BF), gastrocnemius medialis (GM) } \\
\text { and tibialis anterior (TA) activations were evaluated during maximal } \\
\text { instep kicks performed with both feet and with the ball in five conditions } \\
\text { (n=18): stationary (STAT), approaching anteriorly (ANT), posteriorly } \\
\text { (POST), laterally (LAT) and medially (MED). A repeated-measures two- } \\
\text { way ANOVA compared muscle activations between feet and ball } \\
\text { conditions throughout the kicking (0-100\%) and follow-through phases } \\
\text { (101-200\%). Close to ball contact (81-124\%), non-dominant support GM } \\
\text { had greater activation compared to the dominant one. The LAT and MED } \\
\text { conditions differed at certain points of the cycle in the swinging VM (0- } \\
\text { 21\%; 191-200\%), BF (13-70\%; 121-161\%), GM (22-82\%; } 121-143 \%) \\
\text { and TA (0-32\%; 55-97\%; } 186-200 \%) \text { and in support VM (0-81\%), BF } \\
\text { (6-24\%; } 121-161 \%) \text { and GM (24-87\%). Players require greater support } \\
\text { GM activation to stabilize the ankle joint during non-dominant kicks. } \\
\text { Furthermore, lower limb muscle activation differences between LAT and } \\
\text { MED indicate that the kicking strategies are altered when kicking balls } \\
\text { approaching from different conditions. }\end{array}$ \\
\hline
\end{tabular}

\section{SCHOLARONE \\ Manuscripts}


1 Lower limbs muscle activation during instep kick in soccer: effects of dominance and ball 2 condition

5 MS RSMF-2020-0260 submitted to Science and Medicine in Football- R1 22 December 2020

6 Conflict of Interest Disclosure: The authors report no conflict of interest

7 Funding: This research did not receive any specific grant from funding agencies in the public, 8 commercial, or not-for-profit sectors. 


\section{Abstract}

11 Muscle activation during soccer kicking has been studied exclusively with players kicking

12 stationary balls with the dominant foot. This study evaluated swinging and support limb muscle

13 activation during the same kind of kick (instep) using different feet and ball approach conditions.

14 Vastus medialis (VM), biceps femoris (BF), gastrocnemius medialis (GM) and tibialis anterior

15 (TA) activations were evaluated during maximal instep kicks performed with both feet and with

16 the ball in five conditions $(\mathrm{n}=18)$ : stationary (STAT), approaching anteriorly (ANT), posteriorly

17 (POST), laterally (LAT) and medially (MED). A repeated-measures two-way ANOVA compared

18 muscle activations between feet and ball conditions throughout the kicking (0-100\%) and follow-

19 through phases (101-200\%). Close to ball contact (81-124\%), non-dominant support GM had

20 greater activation compared to the dominant one. The LAT and MED conditions differed at certain

21 points of the cycle in the swinging VM (0-21\%; 191-200\%), BF (13-70\%; 121-161\%), GM (22-

$2282 \% ; 121-143 \%)$ and TA $(0-32 \% ; 55-97 \% ; 186-200 \%)$ and in support VM (0-81\%), BF (6-24\%;

23 121-161\%) and GM (24-87\%). Players require greater support GM activation to stabilize the ankle

24 joint during non-dominant kicks. Furthermore, lower limb muscle activation differences between

25 LAT and MED indicate that the kicking strategies are altered when kicking balls approaching from

26 different conditions.

27

28 Keywords: kicking, EMG, moving ball, sports motion

29

30 Word count: 3477 


\section{Introduction}

Kicking is among the most important actions in soccer, allowing players to move the ball with precision, passing to better-positioned teammates trying to score. However, it is a dynamic movement that requires high levels of power and coordination ${ }^{1}$. When kicking, players must position their support foot at an appropriate distance from the ball, accelerate the thigh and shank segments of the swinging leg and reach maximum foot speed near ball contact. ${ }^{2,3}$ To be successful, all involved muscles must be activated with correct timing and magnitude. At the swinging limb, muscles acting at the knee and ankle joints must work together to accelerate the shank and efficiently contact the ball and then stop it during follow-through, ${ }^{4,5}$ while the support limb muscles position the body's center of mass at an optimal point.

Although kicking is a multi-plane task, most of the movement occurs in the sagittal plane, with the vastus medialis (VM), biceps femoris (BF), gastrocnemius medialis (GM) and tibialis anterior (TA) muscles being often investigated during instep kicking through surface electromyography (EMG)..$^{5-9}$ Particularly, Scurr et al. ${ }^{6}$ found increased swinging VM activation when accurately instep kicking a ball (with the right foot) at the top-right corner compared to other corners, while Manolopoulos et al. ${ }^{7}$ found increased VM activation after a period of training, observing no differences in swinging BF and support GM and BF. Kellis et al. ${ }^{8}$ found increased support BF activation when starting the approach run from $90_{-}^{\circ}$ or $45^{\circ}$ compared to $0^{\circ}$. Meanwhile, Katis et al. ${ }^{5}$ observed increased TA and BF and decreased gastrocnemius activation when kicking accurately at a high target (vs inaccurate kicks) and decreased TA activation when accurately kicking at a low target. Finally, Cerrah et al. ${ }^{9}$ found a trend of positive correlations between ball velocity and VM activation when performing inside-foot kicks and a negative trend between ball velocity and $\mathrm{BF}$ and GM when outside-foot kicks were performed. Furthermore, since non-contact 
54 actions are responsible for more than half of soccer injuries, ${ }^{10}$ and kicking represents a good 55 portion of them, ${ }^{11}$ other studies have used EMG to better understand the demands of this task on 56 the involved muscles in both the swinging and the support limbs. ${ }^{12-14}$ Particularly, the swinging 57 thigh muscles play a role in protecting the joint from ligament injury and muscle strain, ${ }^{14,15}$ while 58 the support limb muscles deal with forces similar to those of running at $3.8 \mathrm{~m} / \mathrm{s}$, putting additional 59 strain in those muscles and possibly leading to injuries when activation is inadequate. ${ }^{8}$

60 During matches, the situations in which a player kicks vary according to field location, 61 position of other players and from where the ball is approaching. Players often find themselves in 62 situations where the most appropriate kicking action is with the non-dominant foot. Therefore, 63 being able to successfully kick with both feet is highly desirable in soccer. ${ }^{16}$ Furthermore, players 64 mostly kick moving balls. In fact, they rarely kick stationary balls outside of "set pieces" (corners, 65 free kicks and penalties). Kicking a moving ball is more complex because it requires high levels 66 of prospective control, where players must adjust the kicking motion's speed and timing to achieve 67 optimal ball contact. ${ }^{17}$

Despite frequent occurrences of soccer kicks performed with the non-dominant foot and 69 with moving balls, it is unknown how agonist and antagonist muscles activate while performing 70 them. Greater understanding of this topic could help players and coaches to target training, seeking 71 to improve performance and avoid injuries when performing these kicks. Therefore, our goal was 72 to identify differences in activation patterns of selected lower limb muscles, in both swinging and 73 support limbs, when kicking a stationary or a moving ball approaching from four different angles, 74 with both dominant and non-dominant feet. We hypothesized that there would be different 75 activation of the muscles investigated close to the moment of ball contact and immediately after 
76 ball contact, when kicking with the dominant limb and when kicking a stationary ball or

77 approaching from the side, as these conditions seem to be the least challenging.

79 Methods

$80 \quad \underline{\text { Subjects }}$

81 Eighteen young male experienced non-professional soccer players volunteered (9 82 defenders, 6 midfielders, 3 forwards; 10 right-footed, 8 left-footed; Table 1). They participated 83 weekly in at least two training sessions and a match with teams involved in competitions organized 84 by the national federation. Subjects who experienced any severe lower limb injuries in the previous 85 six-months or actual physical pain on the day of data collection were excluded. Dominance was 86 assessed using the Waterloo Footedness Questionnaire-Revised. ${ }^{18}$ Approval from the University's 87 ethics committee was obtained (protocol number: 55/19) and all subjects gave informed written 88 consent after having the protocol explained and any questions about the benefits and risks of the 89 study answered.

$91 \quad$ Experimental procedures

Subjects wore tight-fitting shorts and indoor soccer shoes. Passive reflective markers were 93 positioned on the ball and on both shoes in correspondence to the calcaneus and the second and 94 fifth metatarsal heads. After careful skin preparation (shaving and cleaning with alcohol), disc95 shaped silver-silver chloride bipolar (diameter: $24 \mathrm{~mm}$; interelectrode distance: $2 \mathrm{~cm}$; Covidien, 96 Dublin, Ireland) were placed over the muscle bellies and aligned with expected muscle fiber 97 orientation of the VM, BF, GM and TA of both limbs. ${ }^{19}$ FreeEMG 300 (BTS S.p.A, Garbagnate 98 Milanese, Italy), with a sampling rate of $1000 \mathrm{~Hz}$, 16bit resolution and differential amplifiers 
99 (bandwidth: 10-500 Hz) with common mode rejection ratio $>110 \mathrm{~dB}$ at $50-60 \mathrm{~Hz}$ and an input

100

101

102

103

104

105

106

107

108

109

110

111

112

113

114

115

116

117

118

119 impedance $>10 \mathrm{G} \Omega$ was used to record muscle activation.

The laboratory was equipped with artificial turf and a nine-camera optoelectronic motion analyzer, sampling at $60 \mathrm{~Hz}$ (BTS S.p.A, Garbagnate Milanese, Italy). Players warmed-up for five minutes on a stationary bike and with ten minutes of soccer-specific exercises. After performing the desired amount of practice trials, subjects were instructed to kick a stationary ball (5-size FIFA approved) as fast as possible into a net to find the maximal functional activation of the selected muscles. ${ }^{5,7}$ Three maximum trials were performed with each foot. All kicks were performed with the instep portion of the foot, to achieve maximal ball speed..$^{9,20}$

\section{$<<$ FIGURE 1 $>>$}

Next, volunteers performed three kicks with the ball in each of the following conditions: (1) stationary (STAT), rolling ball approaching from the subject's (2) anterior side (ANT), (3) posterior side (POST), (4) perpendicular from the kicking foot's lateral side (LAT), (5) perpendicular from the kicking foot's medial side (MED). Participants were asked to kick the ball as fast as possible, starting the run at a $45^{\circ}$ angle two meters from the kicking point, hitting a target $(1.2 \mathrm{~m} \times 1.2 \mathrm{~m})$ in a net positioned three meters away. Figure 1 depicts the starting positions during

the protocol. All kicks were performed with the dominant and non-dominant feet and the order of conditions and feet were randomized between subjects. Three valid trials (hitting the target and contacting ball with foot's instep portion) were recorded for each kick condition (30-second rest).

During rolling ball trials, the ball was released from a small ramp positioned two meters away from the kicking point (average speed: $2 \mathrm{~m} / \mathrm{s}$ ). 
120

121

122

123

124

125

126

127

128

129

130

131

132

133

134 135 condition.

$136 \quad$ Statistical analysis

137

138

139

140

141

142

$\underline{\text { Data processing }}$

\section{Statistical analysis}

Kicks were divided into two phases based on three events: kicking phase (from swinging toe-off until ball contact) and follow-through phase (from ball contact until maximum foot height) (Figure 2). Events were manually identified through visual inspection of the video in which the position of the reflective markers placed on the shoes and on the ball could be observed during kicking, which was synchronized with EMG data. Average phases duration and total kick time were measured for all kicks.

\section{$<<$ FIGURE 2>>}

Electromyographical data were rectified and filtered using a $4^{\text {th }}$ order Butterworth filter (high-pass: $20 \mathrm{~Hz}$; low-pass: $6 \mathrm{~Hz}$ ). Peak muscle activation for all the eight muscles was obtained from the maximum kicks and used for normalization. Normalization using peak EMG values during ballistic tasks has been shown to be highly reliable for all four muscles evaluated in this study ${ }^{21}$ and has been previously used in kicking EMG studies..$^{5,7}$ Each phase was resampled on 100 time points, thus muscle activation during the kicking and follow-through phases was described by a 200-samples curve. These time series were averaged between the three trials of each

G*Power (version 3.1.9.6; University of Trier, Trier, Germany) was used to calculate the appropriate a priori sample size. A repeated-measures ANOVA, within-between interaction (F tests family) was used, with significance level set at $p=.05$ and power set at 0.95 to detect medium effects $(\mathrm{f}>0.35)$. A minimum of 18 subjects was required.

Prior to all analyses, a Shapiro-Wilk test confirmed that data were normally distributed. A two-way $(5 \times 2)$ ANOVA was performed to measure differences between the factors: condition 
143 and foot.For duration, average phases and total kick time were compared and partial eta squared $144\left(\eta_{p}{ }^{2}\right)$ was calculated, while for muscle activation the kicking cycles time series was compared using 145 Statistical Parametric Mapping (SPM). Bonferroni post-hoc tests explored eventual differences 146 between conditions. SPM allows the analysis of the whole movement cycles, drawing conclusions 147 based on the behavior of the variable through time, avoiding loss of information due to 148 discretization. ${ }^{22}$ All statistical analyses were performed using MATLAB (Version R2020a; 149 Mathworks Inc., Natwick, USA).

150 


\section{Results}

152

$<<$ TABLE 2>>

In the swinging VM (Figure 3A), at the beginning of the kicking phase (from 0 to $21 \%$ ) and at 157 the end of follow-through (191 to 200\%), MED condition had higher activation than LAT. 158 Swinging BF (Figure 3B) activation was higher in MED than in LAT throughout most of the 159 kicking phase (from 13 to 70\%), while LAT was higher than MED during part of the follow160 through phase (from 121 to $161 \%$ ).

For swinging GM (Figure 4A), MED had higher activation than LAT for most of the 162 kicking phase (from 22 to $82 \%$ ), while it had lower activation during part of the follow-through 163 (from 121 to $143 \%$ ). Swinging TA activation (Figure 4B) was higher in MED than in LAT in both 164 the kicking phase (from $0 \%$ to $32 \%$ and $55 \%$ to $97 \%$ ) and at the end of follow-through (from $186 \%$ 165 to $200 \%)$

Support VM (Figure 5A) activation was lower in MED than in LAT for most of the kicking 169 phase (from 0 to $81 \%$ ), without differences at ball contact and follow-through. In the support BF 170 (Figure 5B), there was lower MED activation in the kicking phase (from 6 to $74 \%$ ) and higher 171 MED activation during follow-through (from 121 to 161\%) when compared to LAT.

172 In the support GM (Figure 6A), MED presented lower activation than LAT in the kicking 173 phase (from 24 to 87\%). Support TA presented no differences between conditions (Figure 6B). 
Differences between feet were found exclusively in support GM, from $81 \%$ to $124 \%$ of the

175 cycle (Figure 6A). When kicking with the dominant foot, support GM presented lower peak

176 activation midway through the kicking phase, which rapidly decreased, plateauing in the beginning

177 of follow-through. Meanwhile, non-dominant foot kicks presented a delay in peak activation,

178 which was followed by a much slower decrease in activation throughout the kicking cycle.

179 Furthermore, there was an interaction between the foot and condition factors close to ball contact

180 (from 82 to $125 \%$ ), where the MED condition did not seem to present differences between feet.

181

182

183

184

185

186

187

188

189

190

191

192

193

194

195

196

197
$<<$ FIGURE 5>>

$<<$ FIGURE 6>>

\section{Discussion}

Kicking balls approaching from different angles and with both feet are necessary skills in competitive soccer, and players may need to use different muscle activation strategies to perform them successfully. We found that soccer players presented significant differences in the activation of four swinging limb muscles (VM, BF, GM and TA) between LAT and MED conditions in both the kicking and the follow-through phase, while no differences were found between feet. In the support limb, significant differences were found between LAT and MED in the kicking phase for the VM, BF and GM muscles and in the follow-through phase for BF, while non-dominant GM activation was higher close to ball impact. These differences, however, did not seem to affect kicking phase durations.

The kicking duration results suggest that players didn't need to adjust speed to correctly execute different kicks. These findings agree with previous studies that found no differences in kicking duration between feet when performing a drive kick $^{23}$ and a side-foot kick. ${ }^{24}$ However, our results contrast with Egan et al., ${ }^{17}$ who found that the duration of parts of the kicking movement 
198 was shorter when kicking a stationary compared to a moving ball. This difference can be explained 199 by the different events chosen to define the kick and by the slower approaching ball speed used in 200 our study $(2 \mathrm{~m} / \mathrm{s}$ vs $3 \mathrm{~m} / \mathrm{s})$.

201 Between conditions, differences were found in all muscles, except for support-side TA, 202 occurring exclusively between MED and LAT. These results can probably be explained by the 203 technique of the two kicks, which seem to be the most different between the five conditions. In 204 MED, players let the ball pass through before landing the support foot, so that the ball does not hit 205 it on its way to the kicking spot, while in LAT they can position the foot where they like and still 206 hit the ball with good timing by altering the movement of the swinging limb. Changes in support 207 foot position in relation to the ball are reportedly important when performing a more successful 208 kick (with the dominant foot) compared to a less successful one (with the non-dominant foot). ${ }^{23}$ 209 Moreover, in LAT, players face the ball while it approaches, while in MED they must turn their 210 head to follow it. These changes in gaze fixation may lead to postural changes between 211 conditions, ${ }^{25,26}$ affecting the kicking pattern. Although the other conditions also had differences in 212 the movement, players didn't seem to require significantly different muscle activation patterns to 213 perform them. In particular, the lack of differences involving the STAT condition is somewhat 214 unexpected, since it is reportedly a more complex task. ${ }^{17}$

215 The swinging VM muscle, which extends the knee joint to accelerate the kicking foot, 216 usually peaks shortly before ball contact. ${ }^{27}$ Although this activation peak was similar between all 217 conditions, we found in MED a higher activation at the beginning of the kicking phase (while the 218 knee is flexing) and at the end of follow-through (when the foot decelerates) when compared to 219 LAT. This behavior is not optimal since VM activation reduces the knee flexion torque which is 220 required at those particular moments. Meanwhile, the swinging BF was more active in MED 
221 compared to LAT through most of the kicking phase, which may have resulted in greater knee

222 flexion prior to the start of the leg forward motion, which occurs at approximately $75 \%$ of the 223 kicking phase. ${ }^{12}$ The BF muscle eccentrically decelerates the swinging foot after ball contact to 224 prevent the knee from hyperextending. We found lower BF activation in the early stages of the 225 follow-through in MED compared to LAT, which could suggest a decreased contribution of this 226 muscle in decelerating the shank after ball contact. However, the other hamstring muscles also 227 highly contribute to this task and the BF also performs other movements around the knee and hip 228 joints, which could contribute to the differences found. These differences between conditions 229 could have been the result of the different demands that each kick requires from the players, with 230 the MED and LAT possibly being the most different among the conditions.

231 The muscles acting on the ankle joint (TA and GM) of the swinging limb control the foot's 232 position in relation to the ball, with greater TA activation resulting in a higher ball trajectory. ${ }^{28}$ 233 During the kicking phase in the MED condition, both TA and GM were more active than in LAT, 234 suggesting that players had a "stiffer" ankle joint when executing this kick.

The support limb plays a very important role in soccer kick performance, positioning the 236 body in the right place to contact the ball at an optimal angle while supporting loads even higher 237 than those of the swinging limb. ${ }^{29}$ When kicking in the MED condition, players had lower support 238 VM, BF and GM activation during most of the kicking phase when compared to LAT. All the 239 differences found in the support limb between MED and LAT can be justified by the different 240 strategies used by players to land the support foot at an optimal position during different kicks. When comparing muscle activation between kicks with the dominant and non-dominant

242 feet, the only muscle that presented any differences during the kicking cycle was the support GM, 243 which had greater activation across the end of the kicking phase and the beginning of the follow- 
244 through when kicking with the non-dominant limb. It peaked at around $60 \%$ of the kicking phase, 245 which corresponds to the moment when the support foot lands and the swinging hip has reached 246 its maximal extension before starting to accelerate forward. ${ }^{12}$ When kicking with the dominant 247 foot, support GM activation decreased rapidly after its peak, reducing at the moment of ball 248 contact. Meanwhile, when kicking with the non-dominant foot, the activation had a higher peak 249 and decreased more slowly, still being high during ball contact and only reaching low values 250 midway into the follow-through phase. This higher activation of the support GM close to ball 251 contact when kicking with the non-dominant foot is probably a mechanism used by players to 252 further stabilize the ankle joint when performing a more challenging movement, since this muscle 253 plays an important stabilizer role in single-leg tasks. ${ }^{30,31}$

254 By knowing how lower limb muscles behave during different kicks or when kicking with 255 different feet, coaches and athletes could develop training plans that improve kicking performance. 256 Furthermore, we developed a protocol to evaluate muscle activation during kicking that is closer 257 to the reality of competitive matches, possibly contributing to the development of normative data, 258 which can ultimately allow sport scientists to compare their players' activation curves shape to 259 those already found in different populations. This comparison can help identify players that have 260 different patterns or activation asymmetries that can put them at risk of injury or result in worse 261 kicking performance. Furthermore, since kicks performed with different feet and conditions result 262 in distinct activation patterns, it could be advantageous to practice in a variety of situations, 263 changing the stimulus as much as possible to adapt players to several in-game kicking situations. 264 We evaluated the activation of four lower limb muscles of experienced soccer players, both 265 of the swinging and the support limbs, throughout the kicking cycle. To the best of our knowledge, 266 no prior study has used EMG to measure the influence of dominance or ball condition on the 
267 kicking movement, having been limited to investigate kicks with a stationary ball performed with 268 the dominant foot.

However, we must acknowledge some limitations. We evaluated only four muscles on each

270 limb, but other muscles, particularly those that produce movement in other planes of movements,

271 also contribute to kicking performance. Although normalization using maximum functional 272 movement is reliable and more ecological than maximal isometric voluntary contractions, ${ }^{5,7,21}$ for 273 this study protocol, it might have mitigated eventual differences between limbs. In addition, 274 activation of some muscles during surface EMG can be affected by crosstalk. ${ }^{32}$ Furthermore, no 275 performance indicators were measured, such as ball velocity, accuracy or perceived kicking 276 difficulty, which could have helped determine if and how different activation patterns alter kicking 277 quality. During competitive matches, an infinite number of combinations of ball angle of approach, 278 distance and speed can be seen, however for this laboratory study, a limited number of conditions 279 was defined. No kinematic measurements were performed during the kicks, which didn't allow us 280 to divide the kicking phase in further sub-phases ${ }^{12,33}$ and to associate the position of the subjects' lower limbs with the corresponding muscle activation at any given point. Finally, our sample was composed of adult male soccer players, however, different populations (females, younger subjects) may display different approaches to respond to the conditions presented. In conclusion, we investigated the activation differences of the VM, BF, GM and TA 285 muscles when the instep kick is performed in different conditions with both feet. We found that 286 the support GM has an earlier activation peak and a lower activation magnitude near ball contact 287 when kicking the ball with the dominant foot. We also found that all muscles investigated (except 288 the support TA) had differences during the cycle when comparing the LAT to the MED conditions, 289 but without any differences when comparing to the other conditions (STAT, ANT and POST). 
290 Coaches should incorporate different angles of ball approach and kicks with the non-dominant foot 291 in their practices, since there are several differences in muscle activation patterns between 292 conditions and feet. Ultimately, this can lead to players being better prepared to perform these 293 tasks during in-game situations. Future studies should perform similar protocols, measuring 294 activation of more muscles combined with kinematic data in order to provide more specific 295 recommendations to improve kicking performance. 
297

298

299

300

301

302

303

304

305

306

307

308

309

310

311

312

313

314

315

316

317

318

319

320

321

322

323

324

325

326

\section{References}

1. Naito K, Fukui Y, Maruyama T. Multijoint kinetic chain analysis of knee extension during the soccer instep kick. Hum Mov Sci. 2010;29(2):259-276.

2. Nunome H, Ikegami Y, Kozakai R, Apriantono T, Sano S. Segmental dynamics of soccer instep kicking with the preferred and non-preferred leg. J Sports Sci. 2006;24(5):529-541.

3. Palucci Vieira LH, Barbieri FA, Kellis E, et al. Organisation of instep kicking in young U11 to U20 soccer players. Sci Med Footb. Published online 2020.

4. Severin AC, Mellifont DB, Sayers MGL. Influence of previous groin pain on hip and pelvic instep kick kinematics. Sci Med Footb. 2017;1(1):80-85.

5. Katis A, Giannadakis E, Kannas T, Amiridis I, Kellis E, Lees A. Mechanisms that influence accuracy of the soccer kick. J Electromyogr Kinesiol. 2013;23(1):125-131.

6. Scurr JC, Abbott V, Ball N. Quadriceps EMG muscle activation during accurate soccer instep kicking. J Sports Sci. 2011;29(3):247-251.

7. Manolopoulos E, Papadopoulos C, Kellis E. Effects of combined strength and kick coordination training on soccer kick biomechanics in amateur players. Scand J Med Sci Sport. 2006;16(2):102-110.

8. Kellis E, Katis A, Gissis I. Knee biomechanics of the support leg in soccer kicks from three angles of approach. Med Sci Sports Exerc. 2004;36(6):1017-1028.

9. Cerrah AO, Soylu AR, Ertan H, Lees A. The effect of kick type on the relationship between kicking leg muscle activation and ball velocity. Montenegrin J Sport Sci Med. 2018;7(1):3944.

10. Hawkins RD, Fuller CW. A prospective epidemiological study of injuries in four English professional football clubs. Br J Sports Med. 1999;33(3):196-203.

11. Rahnama N, Reilly $\mathrm{T}$, Lees A. Injury risk associated with playing actions during competitive soccer. Br J Sports Med. 2002;36(5):354-359.

12. Brophy RH, Backus SI, Pansy BS, Lyman S, Williams RJ. Lower extremity muscle activation and alignment during the soccer instep and side-foot kicks. J Orthop Sports Phys Ther. 2007;37(5):260-268.

13. Brophy RH, Backus S, Kraszewski AP, et al. Differences between sexes in lower extremity alignment and muscle activation during soccer kick. J Bone Jt Surg - Ser A. 2010;92(11):2050-2058. 
327 14. Cordeiro N, Cortes N, Fernandes O, Diniz A, Pezarat-Correia P. Dynamic knee stability 328 and ballistic knee movement after ACL reconstruction: an application on instep soccer kick. 329 Knee Surgery, Sport Traumatol Arthrosc. 2015;23(4):1100-1106.

330 15. Opar DA, Williams MD, Shield AJ. Hamstring strain injuries: Factors that Lead to injury 331 and re-Injury. Sport Med. 2012;42(3):209-226.

332 16. Carey DP, Smith G, Smith DT, et al. Footedness in world soccer: An analysis of France ' 98. $333 \quad J$ Sports Sci. 2001;19(11):855-864.

334 17. Egan CD, Verheul MHG, Savelsbergh GJP. Effects of experience on the coordination of 335 336 337 338 339 340 341 internally and externally timed soccer kicks. $J$ Mot Behav. 2007;39(5):423-432.

18. Elias LJ, Bryden MP, Bulman-Fleming MB. Footedness is a better predictor than is handedness of emotional lateralization. Neuropsychologia. 1998;36(1):37-43.

19. Hermens HJ, Freriks B, Disselhorst-Klug C, Rau G. Development of recommendations for SEMG sensors and sensor placement procedures. J Electromyogr Kinesiol. 2000;10(5):361374.

20. Lees A, Nolan L. The biomechanics of soccer: A review. J Sports Sci. 1998;16(3):211-234.

21. Suydam SM, Manal K, Buchanan TS. The advantages of normalizing electromyography to ballistic rather than isometric or isokinetic tasks. J Appl Biomech. 2017;33(3):189-196.

22. Pataky TC. Generalized n-dimensional biomechanical field analysis using statistical parametric mapping. J Biomech. 2010;43(10):1976-1982.

23. McLean BD, Tumilty DMA. Left-right asymmetry in two types of soccer kick. Br J Sports Med. 1993;27(4):260-262.

24. Zago M, Motta AF, Mapelli A, Annoni I, Galvani C, Sforza C. Effect of Leg Dominance on The Center-of-Mass Kinematics During an Inside-of-the-Foot Kick in Amateur Soccer Players. J Hum Kinet. 2014;42(1):51-61.

25. Raffi M, Piras A, Persiani M, Perazzolo M, Squatrito S. Angle of gaze and optic flow direction modulate body sway. J Electromyogr Kinesiol. 2017;35:61-68.

26. Fiorelli CM, Polastri PF, Rodrigues ST, et al. Gaze position interferes in body sway in young adults. Neurosci Lett. 2017;660(November 2016):130-134.

27. Cerrah AO, Gungor EO, Soylu AR, Ertan H, Lees A, Bayrak C. Muscular activation patterns during the soccer in-step kick. Isokinet Exerc Sci. 2011;19(3):181-190.

28. Katis A, Giannadakis E, Kannas T, Amiridis I, Kellis E, Lees A. Mechanisms that influence 
358 accuracy of the soccer kick. J Electromyogr Kinesiol. 2013;23(1):125-131.

359 29. Lees A, Asai T, Andersen TB, Nunome H, Sterzing T. The biomechanics of kicking in soccer: A review. J Sports Sci. 2010;28(8):805-817.

361 30. Delahunt E, Monaghan K, Caulfield B. Changes in lower limb kinematics, kinetics, and 362 muscle activity in subjects with functional instability of the ankle joint during a single leg 363 drop jump. J Orthop Res. 2006;24(10):1991-2000.

364 31. Kim H, Palmieri-Smith R, Kipp K. Time-frequency analysis of muscle activation patterns 365 in people with chronic ankle instability during Landing and cutting tasks. Gait Posture. $366 \quad 2020 ; 82: 203-208$.

367 32. Wong YM, Straub RK, Powers CM. The VMO: VL activation ratio while squatting with 368 hip adduction is influenced by the choice of recording electrode. J Electromyogr Kinesiol. $369 \quad 2013 ; 23(2): 443-447$.

370 33. Nunome H, Asai T, Ikegami Y, Sakurai S. Three-dimensional kinetic analysis of side-foot $371 \quad$ and instep soccer kicks. Med Sci Sports Exerc. 2002;34(12):2028-2036. 
373 Table 1. Demographics and anthropometrics of the participants.

\begin{tabular}{lccc}
\hline & Mean & SD & Range \\
\hline Age (years) & 21.1 & 2.4 & $19-28$ \\
Weight (kg) & 70.8 & 6.2 & $60-83$ \\
Height (cm) & 177.4 & 5.5 & $167-186$ \\
Experience (years) & 14.4 & 2.6 & $10-20$ \\
Training sessions/week (n) & 2.6 & 0.8 & $2-5$ \\
\hline
\end{tabular}

374

375 
376 Table 2. Kick phase duration and total kick time. Values are presented as mean and standard 377 deviation (mean \pm SD).

\begin{tabular}{|c|c|c|c|}
\hline & KICKING (s) & $\begin{array}{c}\text { FOLLOW- } \\
\text { THROUGH (s) }\end{array}$ & TOTAL (s) \\
\hline CONDITION & & DOMINANT & \\
\hline STAT & $0.23 \pm 0.03$ & $0.16 \pm 0.02$ & $0.39 \pm 0.05$ \\
\hline ANT & $0.23 \pm 0.03$ & $0.16 \pm 0.02$ & $0.39 \pm 0.05$ \\
\hline POST & $0.23 \pm 0.02$ & $0.18 \pm 0.03$ & $0.41 \pm 0.03$ \\
\hline LAT & $0.23 \pm 0.03$ & $0.18 \pm 0.06$ & $0.41 \pm 0.07$ \\
\hline MED & $0.23 \pm 0.03$ & $0.17 \pm 0.04$ & $0.40 \pm 0.04$ \\
\hline CONDITION & 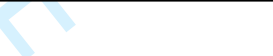 & NON-DOMINANT & \\
\hline STAT & $0.24 \pm 0.03$ & $0.18 \pm 0.04$ & $0.41 \pm 0.06$ \\
\hline ANT & $0.24 \pm 0.02$ & $0.17 \pm 0.04$ & $0.41 \pm 0.05$ \\
\hline POST & $0.24 \pm 0.02$ & $0.18 \pm 0.03$ & $0.41 \pm 0.03$ \\
\hline LAT & $0.23 \pm 0.03$ & $0.16 \pm 0.04$ & $0.39 \pm 0.07$ \\
\hline MED & $0.23 \pm 0.02$ & $0.18 \pm 0.04$ & $0.41 \pm 0.04$ \\
\hline \multicolumn{4}{|l|}{ p values } \\
\hline Condition & 0.513 & 0.345 & 0.170 \\
\hline Feet & 0.084 & 0.577 & 0.228 \\
\hline Interaction & 0.328 & 0.068 & 0.122 \\
\hline \multicolumn{4}{|c|}{ Partial eta squared $\left(\eta_{p}^{2}\right)$} \\
\hline Condition & 0.041 & 0.061 & 0.098 \\
\hline Feet & 0.166 & 0.019 & 0.084 \\
\hline Interaction & 0.065 & 0.150 & 0.100 \\
\hline
\end{tabular}

Ball approach: STAT: Stationary; ANT: Anterior; POST: Posterior; LAT: Lateral from kicking 379 foot; MED: Medial from kicking foot. 
381 Figure 1 - Diagram showing the positions in which the ball and the subjects started in relation to 382 the kicking point. When the ball comes from the sides the condition changes whether the subject 383 is kicking with the right or left foot.

384 385 386

Figure 2 - Illustration of kicking events and relevant phases.

Figure 3 - Swinging vastus medialis (A) and biceps femoris (B) activation during kicking (1100) and follow-through (101-200) phases. Curves are mean \pm SD of all participants' trials.

Figure 4 - Swinging gastrocnemius medialis (A) and tibialis anterior (B) activation during kicking (1-100) and follow-through (1-100) phases. Curves are mean \pm SD of all participants' trials.

Figure 5 - Support vastus medialis (A) and biceps femoris (B) activation during kicking (1-100) and follow-through (101-200) phases. Curves are mean \pm SD of all participants' trials.

Figure 6 - Support gastrocnemius medialis (A) and tibialis anterior (B) activation during kicking 400 
Figure 1 - Diagram showing the positions in which the ball and the subjects started in relation to the kicking point. When the ball comes from the sides the condition changes whether the subject is kicking with the right or left foot.

$254 \times 190 \mathrm{~mm}(96 \times 96 \mathrm{DPI})$ 


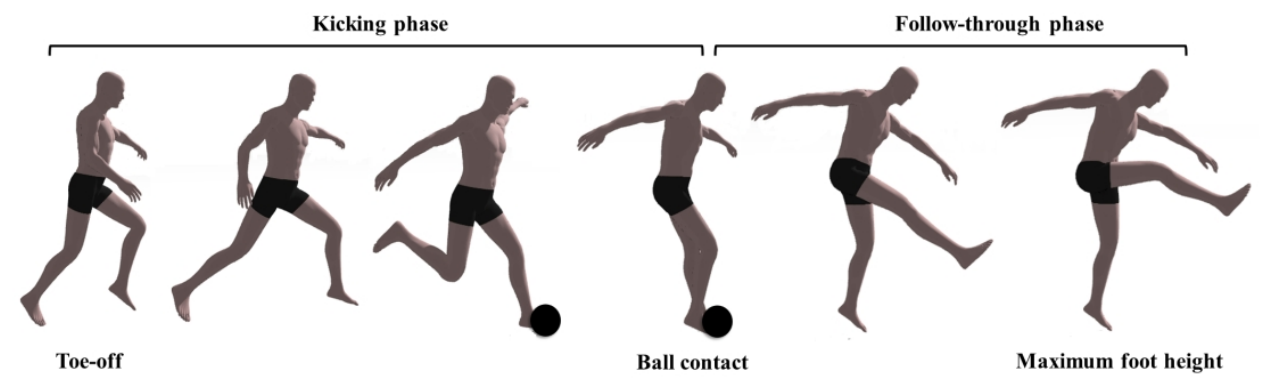

Figure 2 - Illustration of kicking events and relevant phases. 
SWINGING THIGH

Dominant foot

A
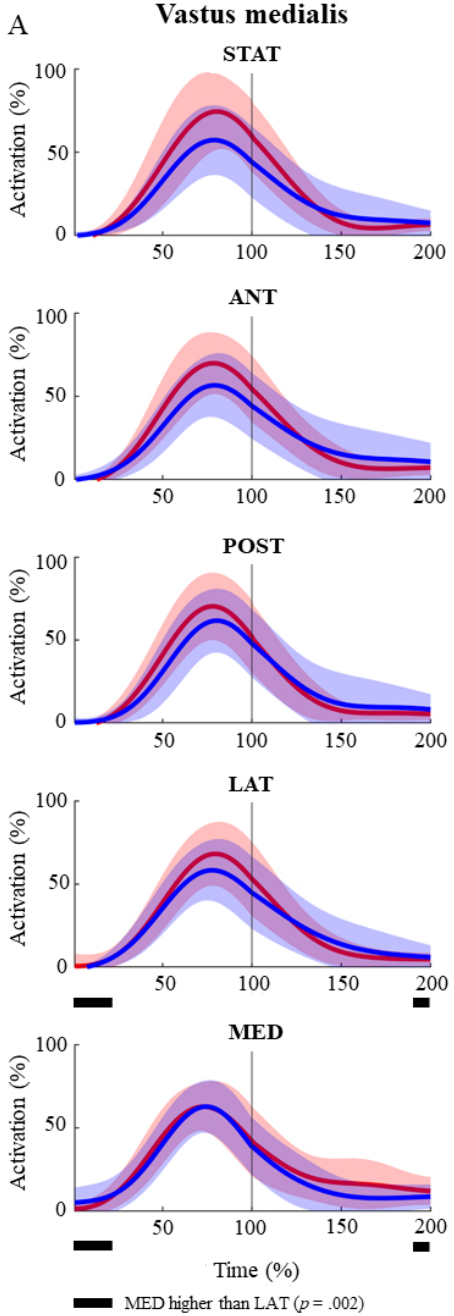

\begin{tabular}{l|l} 
Non-dominant foot & Ball contact
\end{tabular}
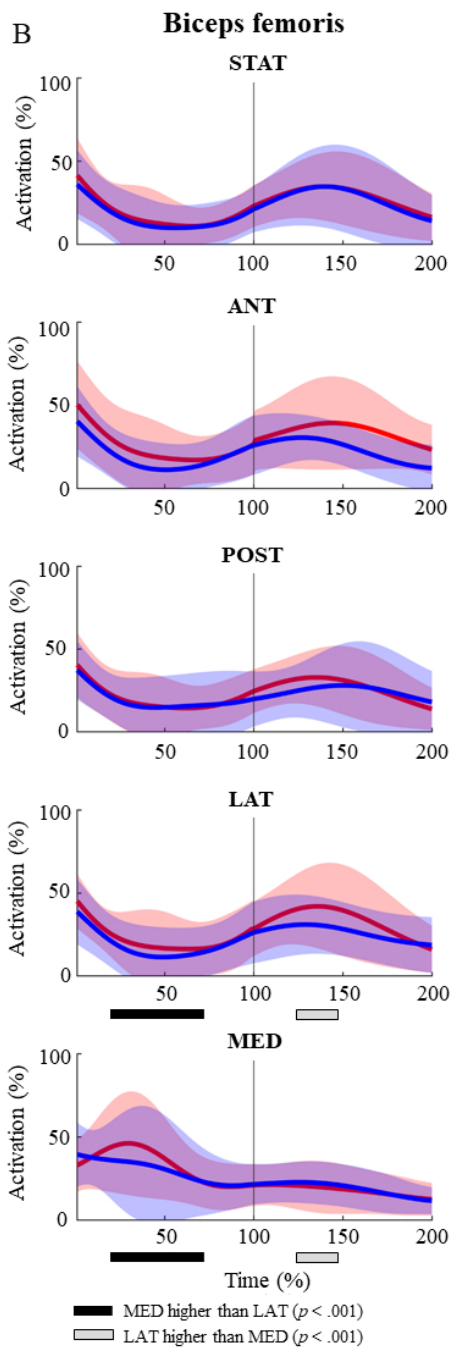

Figure 3 - Swinging vastus medialis (A) and biceps femoris (B) activation during kicking (1-100) and followthrough (101-200) phases. Curves are mean \pm SD of all participants' trials.

\section{$228 \times 338 \mathrm{~mm}(96 \times 96 \mathrm{DPI})$}


SWINGING SHANK

Dominant foot $\quad$ Non-dominant foot $\quad$ Ball contact
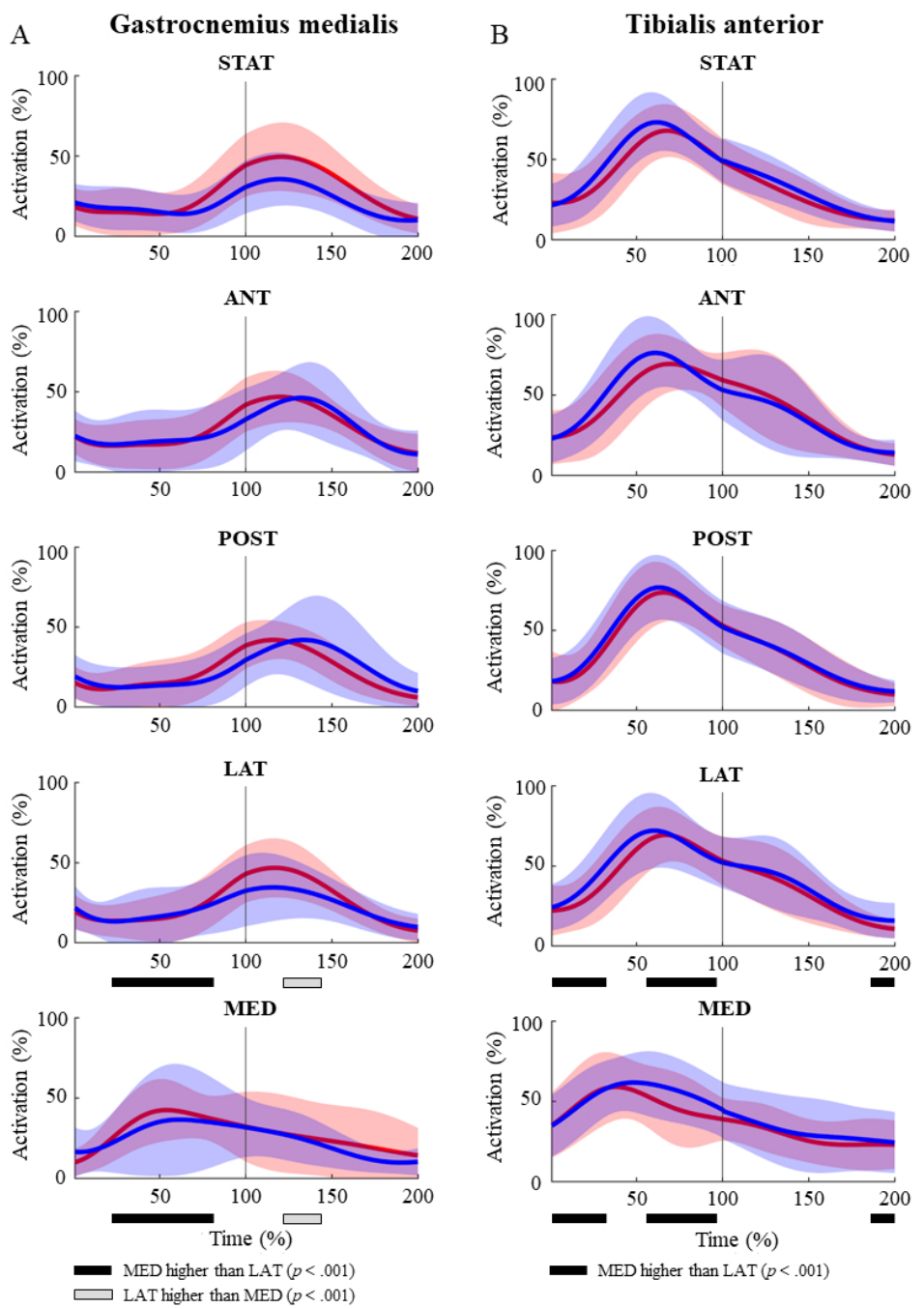

Figure 4 - Swinging gastrocnemius medialis (A) and tibialis anterior (B) activation during kicking (1-100) and follow-through (1-100) phases. Curves are mean \pm SD of all participants' trials.

\section{$228 \times 338 \mathrm{~mm}(96 \times 96 \mathrm{DPI})$}


SUPPORT THIGH

Dominant foot

A
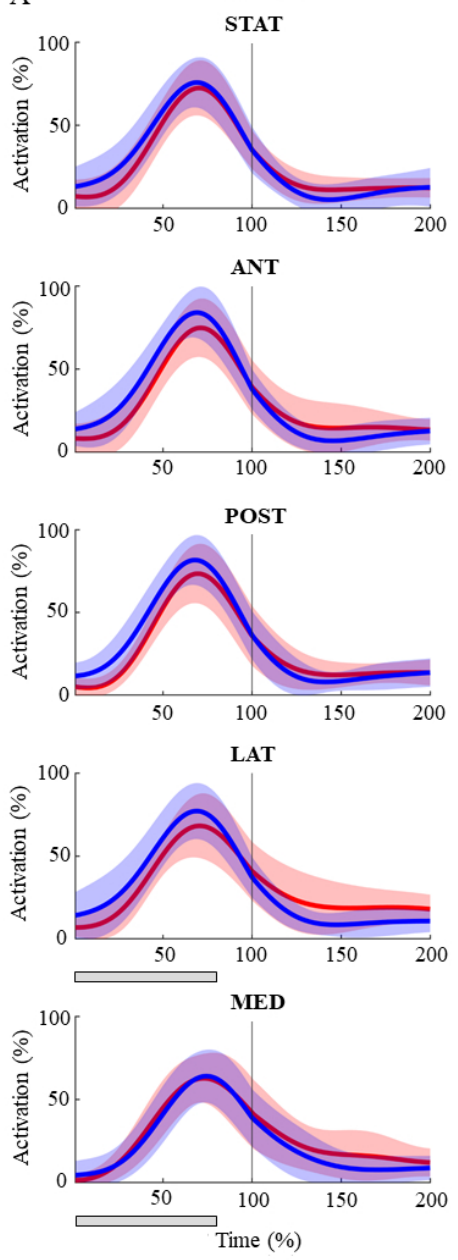

$\square$ LAT higher than MED $(p<.001)$
Non-dominant foot
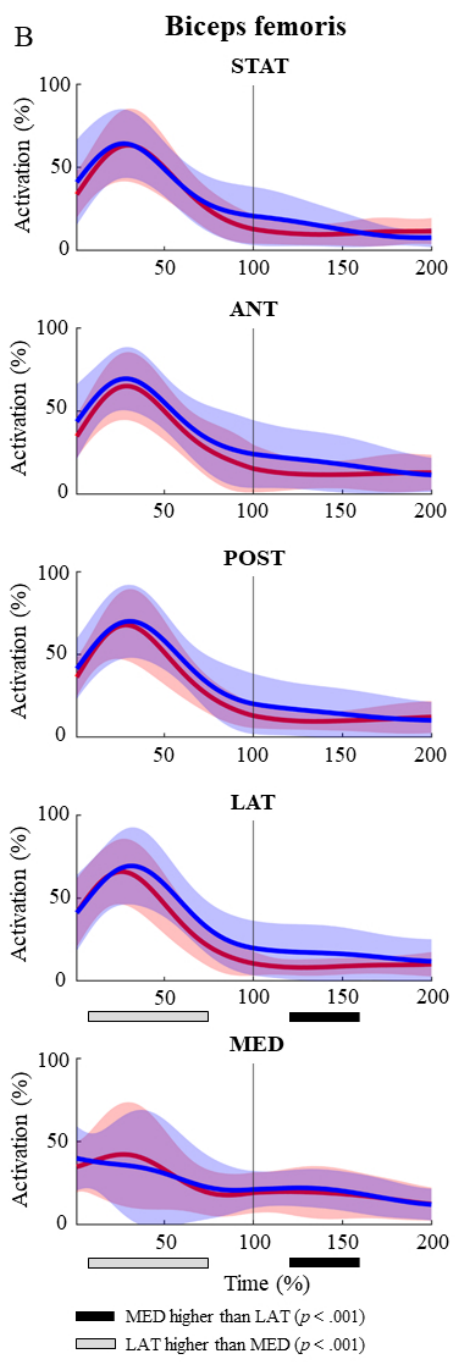

Figure 5 - Support vastus medialis (A) and biceps femoris (B) activation during kicking (1-100) and followthrough (101-200) phases. Curves are mean \pm SD of all participants' trials.

\section{$228 \times 338 \mathrm{~mm}(96 \times 96 \mathrm{DPI})$}


SUPPORT SHANK

Dominant foot

A
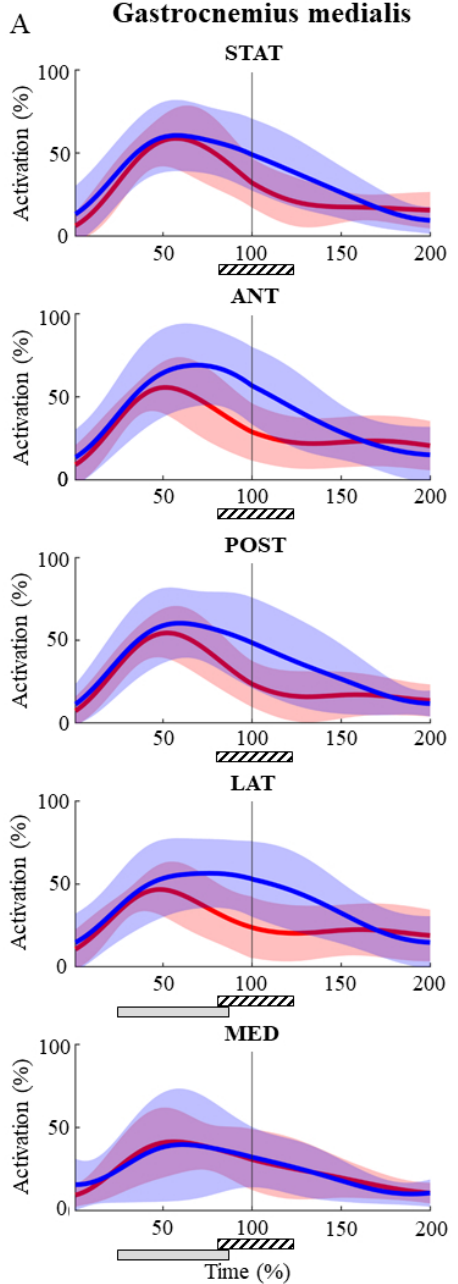

$\square$ MED higher than LAT $(p<.001)$

ZIZ Non-dominant higher than dominant $(p<.001)$
Non-dominant foot $\quad$ Ball contact
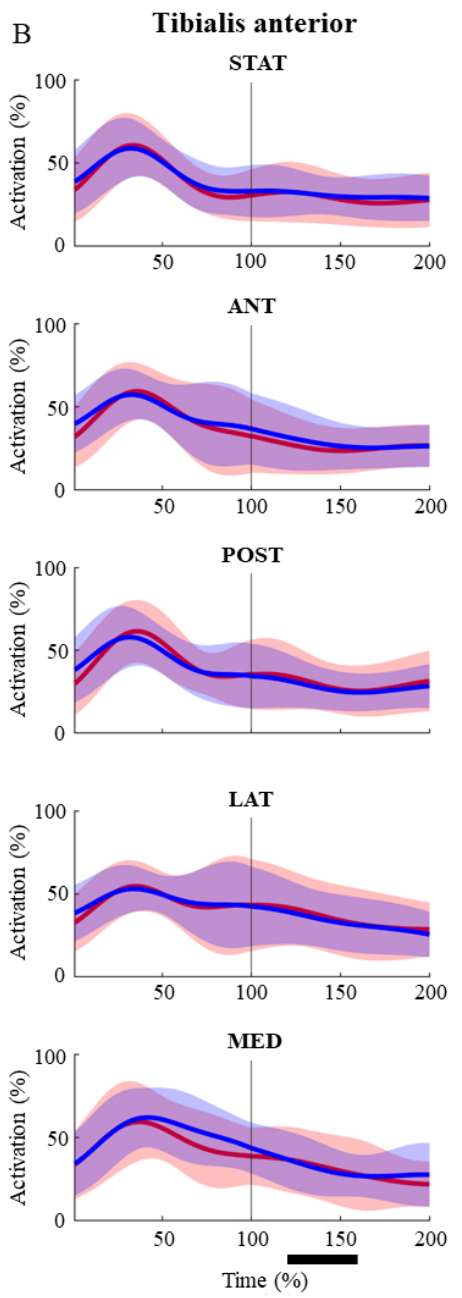

Figure 6 - Support gastrocnemius medialis (A) and tibialis anterior (B) activation during kicking (1-100) and follow-through (101-200) phases. Curves are mean \pm SD of all participants' trials.

\section{$228 \times 338 \mathrm{~mm}(96 \times 96 \mathrm{DPI})$}

